\title{
Granulation tissue enriched by aspirin and omega-3 fatty acids in healing experimental periodontal lesion
}

\author{
Filip Hromcik ${ }^{\mathrm{a}, \mathrm{b}}$, Jan Vokurka ${ }^{\mathrm{a}, \mathrm{b}, \mathrm{t}}$, Eduard Gopfert ${ }^{c}$, Martin Faldyna ${ }^{c}$, Marketa Hermanova ${ }^{\mathrm{b}, \mathrm{d}, \mathrm{t},}$, Michal Kyr $^{\mathrm{b}, \mathrm{e}, \mathrm{t}}$, \\ Monika Vicenovac,", Lydie Izakovicova Hollaa,
}

\begin{abstract}
Aims. Granulation tissue (GT) and specialized pro-resolving mediators such as lipoxins and resolvins are key elements in the successful resolution of periodontitis. Aspirin-triggered lipoxins and resolvins are even more powerful than their natural analogues. Their biosynthesis can be accelerated by omega-3 fatty acids. The aim of this study was to evaluate the use of GT enriched by aspirin and omega-3 fatty acids during the surgical treatment of periodontitis in an experimental animal model (rabbit).

Methods. In each of 24 rabbits, two experimental periodontal defects were created. In total, 47 defects were treated with open-flap debridement and one of three procedures: (1) GT extracted and soaked with aspirin and omega-3 fatty acids (ASA+OMEGA3 group); (2) GT soaked with saline (PLACEBO group); or (3) GT left untreated (CONTROL group). Then, the GT was replaced in situ. Primary evaluated criteria were the probing pocket depth (PPD) and the clinical attachment level (CAL). Necropsies were harvested 2, 6, and 12 weeks after surgery. The samples were used for histological and molecular biological assessment.

Results. A trend of greater PPD and CAL in the ASA+OMEGA3 group was observed at 6 weeks. However, there was no significant difference between them. During the observation period, tissue levels of FGF-7, IL-1 $\beta$ and TIMP-1 showed a statistically significant decrease $(P<0.05)$. For the other variables, the ASA+OMEGA3 group was comparable with the PLACEBO and CONTROL groups.

Conclusion. This experiment did not demonstrate the superiority of the proposed approach. However, the enriched granulation tissue did not impair healing outcomes.
\end{abstract}

Key words: periodontitis, granulation tissue, oral surgery, inflammation mediators, lipoxin

Received: November 3, 2019; Revised: December 23, 2019; Accepted: January 22, 2020; Available online: February 11, 2020

https://doi.org/10.5507/bp.2020.003

(c) 2021 The Authors; https://creativecommons.org/licenses/by/4.0/

${ }^{a}$ Clinic of Dentistry, St. Anne's Faculty Hospital, Pekarska 53, 656 91, Brno, Czech Republic

${ }^{b}$ Faculty of Medicine, Masaryk University, Kamenice 5, 625 00, Brno, Czech Republic

'Veterinary Research Institute, Hudcova 70, 621 00, Brno, Czech Republic

${ }^{d}$ First Department of Pathology, St. Anne's Faculty Hospital, Pekarska 53, 656 91, Brno, Czech Republic

eDepartment of Pediatric Oncology, University Hospital Brno, Cernopolni 9, 613 00, Brno, Czech Republic

"These authors contributed equally to this work

Corresponding author: Lydie Izakovicova Holla, e-mail: holla@med.muni.cz

\section{INTRODUCTION}

Periodontitis is a multifactorial inflammatory disease that leads to the destruction of periodontal tissues, with potential tooth loss. Its development is associated with bacteria residing in tooth biofilms close to or underneath the gingival margin ${ }^{1}$. Major signs of periodontal inflammation are periodontal pocketing, clinical attachment loss, alveolar bone resorption, and tooth mobility. The treatment of periodontitis lies mainly in eliminating dental biofilm along with calculus from the afflicted tooth surface both above and under the gingival margin and long-term maintenance of a biofilm-free environment ${ }^{2}$.

Until recently, healing of an inflammation, not only periodontitis, was believed to involve a passive process. It has been revealed that in the late stage of inflammation, so-called resolution (termination of inflammation) occurs. The anti-inflammatory pathways are not sufficient for resolution to take place and ruling out the proinflammatory pathways (i.e. with NSAIDs) is not enough for tissues to heal. Resolution has been reported to be an active process leading to the regeneration of tissue and re-establishment of homeostasis ${ }^{3}$.

It has been suggested that periodontitis may be attributable to a lack of adequate resolution of inflammation and hyperactivity of pro-inflammatory cytokines ${ }^{4,5}$. Resolution is driven by a family of lipid molecules known as specialized pro-resolution mediators (SPMs), such as lipoxins and resolvins ${ }^{6}$. These are naturally synthetized in the late phases of inflammation ${ }^{7,8}$. Their synthesis can be triggered by aspirin, which results in more powerful and stable analogues ${ }^{9}$. Both lipoxins and resolvins are derived from poly-unsaturated fatty acids (PUFAs), which are cleaved from the cellular membrane and abundant in fish oil ${ }^{10}$. The acetylation of cyclooxygenase-2 (COX-2) by aspirin not only blocks the production of prostaglan- 
dins and leukotrienes but also switches the activity of this enzyme to acting as a lipoxygenase and, thus, allows the synthesis of lipoxins without cell-to-cell interaction ${ }^{10}$. Additionally, acetylated COX-2 carries out the synthesis of aspirin-triggered resolvins or protectin D1 ( ref. $^{11,12}$ ).

Granulation tissue (GT) possesses great healing capacity and offers a large number of new vessels, fibroblasts and macrophages. Also, periodontal GT was proven to contain a substantial number of mesenchymal stem cells (MSCs), which correlates with its regenerative potential $^{13,14}$. It has been shown that GT promotes regeneration and resolution of inflammation in periodontitis ${ }^{15}$.

Inducing the synthesis of resolvins and lipoxins by the addition of aspirin and PUFAs to GT during open-flap periodontal procedures might help to resolve periodontitis and, thus, enhance, accelerate and shorten periodontal therapy. The aim of the present study was to evaluate the effect of the addition of aspirin and PUFAs to granulation tissue on the healing of experimental periodontitis.

\section{MATERIALS AND METHODS}

\section{Ethical approval}

The experiment was performed in compliance with Act No. 246/1992 Coll. of the Czech National Council for the protection of animals against cruelty and with the agreement of the Branch Commission for Animal Welfare of the Ministry of Agriculture of the Czech Republic.

The study was performed according to the ARRIVE guidelines for reporting in vivo animal research ${ }^{16}$.

\section{Study design}

The study was performed as a controlled blinded trial in an animal model. In twenty-four rabbits, experimental periodontitis was induced via wire ligature and inoculation by $P$. gingivalis. A total of 47 defects were created.
One defect had to be excluded because of a fracture in the first premolar during the experiment. Six weeks after ligature placement, the ligature was removed and the rabbit was subjected to open-flap surgery to remove the granulation tissue (GT) and replace it in its original interdental space after one of the three following procedures: ASA+omega3 group - GT was soaked with fish oil (n-3 PUFAs) and aspirin; PLACEBO group (PL) - GT was soaked with saline; CONTROL group (CO) - GT was left untreated. Necropsies were harvested 2 weeks ( 8 rabbits, 15 defects), 6 weeks ( 6 rabbits, 12 defects) or 12 weeks ( 8 rabbits, 16 defects) after surgery, and the samples were preserved for histological and molecular biological assessments.

\section{Animals}

In this experimental model, twenty-four specificpathogen free 3-month old White New Zealand rabbits (Oryctolagus cuniculus f. domesticus) (supplied by a local production company approved by the Ministry of Agriculture of the Czech Republic and registered as CZ 62760247) were enrolled, including both males and females. They were kept at the Veterinary Research Institute (Brno, Czech Republic) in experimental cages certified by the Ministry of Agriculture of the Czech Republic (CZ 62760124) according to standard protocols corresponding to Czech regulations (The Animal Protection and Welfare Act No. 246/1992 Coll. of the Government of the Czech Republic). The experiments were approved by the Branch Commission for Animal Welfare of the Ministry of Agriculture of the Czech Republic (permission number 22373/2016-MZE-17214).

\section{Ligature-induced periodontitis}

The ligature-induced periodontitis model described in many studies ${ }^{17,18}$ was used to develop periodontal defects. The suspension of Porphyromonas gingivalis used for in-

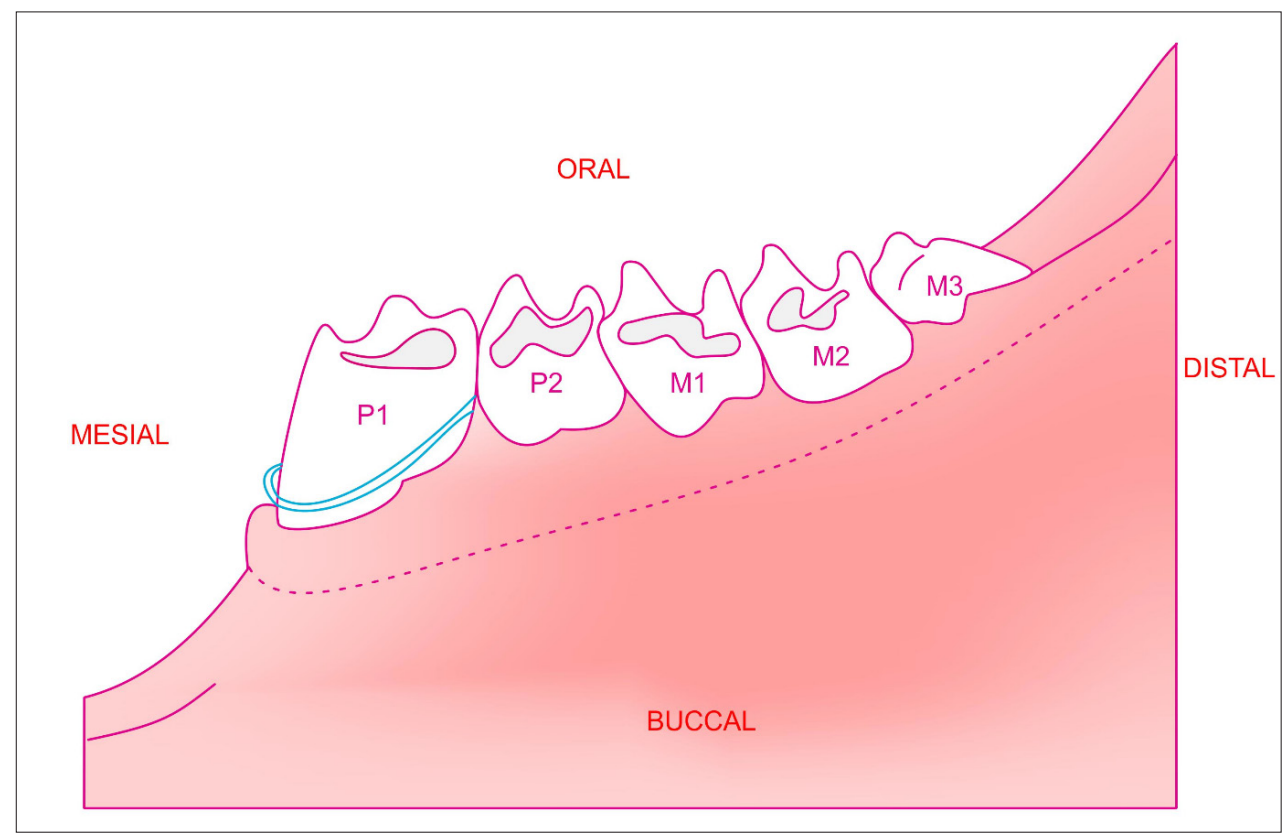

Fig. 1. A ligature was tied around the first mandibular premolar to promote gingival irritation and induce experimental periodontitis. 
duction was cultivated from a stock sample (CCM 3985, isolated from a subcutaneous abscess) and adjusted to the desired optical density (corresponding to a concentration of 4.5-6.5 x10 $10^{8} \mathrm{CFU} / \mathrm{mL}$ ).

Following anaesthesia by intraperitoneal injection of ketamine and xylazine, an orthodontic steel wire of 0.012 inches in diameter was tied around the first mandibular premolars on both the left and right sides as apically as possible (Fig 1). A notch was created in the mesial cervical area of the tooth with a small steel bur to improve retention of the ligature. Moreover, the ligature was secured by applying a small amount of light-cured flow composite after etching the enamel with $37 \%$ phosphoric acid for $30 \mathrm{~s}$. The epithelial junction around the first premolar was disrupted with an elevator. When the ligatures were fastened, two millilitres of $P$. gingivalis suspension was applied to the gingival areas of both lower first premolars. Such inoculation was repeated every day for 6 weeks to establish experimental periodontitis. The ligatures were removed 6 weeks after their placement, and experimental treatment was performed.

\section{Surgery, experimental treatment}

Six weeks after ligature placement, experimental periodontitis had developed, and a second intervention was performed under general anaesthesia, the ligature was removed, and open-flap surgery was performed on the interdental space between the first and second mandibular premolars. The interdental papilla on both buccal and lingual aspects was elevated, and GT was extracted. At this time, a treatment option was randomly chosen for the actual defect. In the ASA+omega 3 group, GT was soaked for four minutes with acetylsalicylic acid at a concentration of $81 \mathrm{mg} / \mathrm{mL}$ (Kardegic, Sanofi Winthrop Industrie, Quétigny, France) and subsequently for another four minutes with an omega-3 PUFA solution (Omega3 Forte, Walmark, Trrinec, Czech Republic) containing 18\% eicosapentaenoic acid (EPA), 12\% docosahexaenoic acid (DHA) and vitamin E. In the PLACEBO group, GT was only soaked with saline. In the CONTROL group, GT was not treated. In all groups, the GT was placed back into

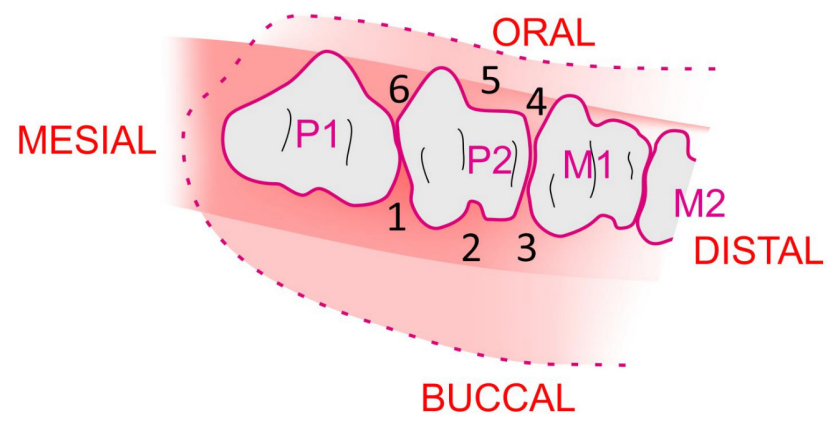

Fig. 2. Measurement of primary observed parameters, probing pocket depth (PPD) and clinical attachment level (CAL). PPD and CAL were measured at six locations around the second mandibular premolar before treatment (day 0 ) and on the day of necropsy ( 2 weeks, 6 weeks or 12 weeks later). its original interdental space, and the papilla was sutured with a single non-resorbable suture (Resolon, Resorba, Nürnberg, Germany). A split-mouth design was followed, so that in a single animal, two defects were treated independently with randomly assigned treatments.

The animals were euthanised 2 weeks ( 8 rabbits, 15 defects), 6 weeks ( 6 rabbits, 12 defects) or 12 weeks ( 8 rabbits, 16 defects) after the surgery (randomly assigned) with a lethal dose of T61 (MSD Animal Health, Boxmeer, Netherlands) applied intracardially under general anaesthesia. Necropsies were then harvested.

\section{Clinical evaluation}

Clinically, the probing pocket depth (PPD) and clinical attachment level (CAL) were measured with UNC periodontal probe (Supplement S9) before surgery and after necropsy at six locations around the second mandibular premolar (Fig. 2) to assess two adjacent interdental spaces - P1-P2 and P2-M1. Clinically, PPD was measured as a distance from the gingival margin to the bottom of the defect or gingival sulcus, respectively. For CAL measurement, the most coronal point of the tooth enamel was used as a reference because no cemento-enamel junction is identifiable in rabbit teeth.

\section{Histological analysis}

Resected specimens of rabbit mandible were processed and histopathologically examined at the $1^{\text {st }}$ Department of Pathology, St. Anne's University Hospital Brno. Resected tissues were initially fixed in $10 \%$ neutral buffered formalin for $48 \mathrm{~h}$ and were subsequently subjected to decalcification in Decalcifier DC1 (LABONORD, Templemars, France) solution containing formic acid and formalin at room temperature. The decalcifying fluid was replaced daily until the completion of decalcification. Tissue slices including the first and second premolar, where the ligature was placed, were used. These specimens were then dehydrated, embedded in paraffin and sectioned along the premolars in a mesiodistal plane. Four-micron-thick tissue sections were stained with haematoxylin-eosin. Mounted slides were histopathologically examined using a uniform microscope and camera settings (Olympus BX43 and PROMICAM 3-12C). The areas between the first and second premolars were analysed under light microscopy. The level of epithelialization of the defects, quality of connective tissue, and presence of inflammatory infiltrates and reactive osteoplasia were evaluated and scored (Table 1). The size of the intrabony defect (distance of the alveolar bone from the top of the defect) was measured using a $100 \mu \mathrm{m}$ scale.

\section{Molecular biological assessment}

RNA isolation and reverse transcription

For RNA stabilization, thirty samples $(<3 \mathrm{~mm})$ were excised from the buccal portion of the interdental papilla P1-P2 during necropsy. The excised tissue was directly immersed in RNAlater (Qiagen, Germany), then kept at $4{ }^{\circ} \mathrm{C}$ 
Table 1. Semi-quantitative classification of histologic parameters used for evaluation of the harvested samples.

\begin{tabular}{|c|c|c|c|c|c|c|c|}
\hline \multicolumn{2}{|c|}{ Level of epithelialization } & \multicolumn{2}{|c|}{ Connective tissue } & \multicolumn{2}{|c|}{ Inflammatory infiltrate } & \multicolumn{2}{|c|}{ Reactive osteoplasia } \\
\hline 1 & Persistent defect & 1 & $\begin{array}{l}\text { Immature, cellular, } \\
\text { granulation tissue }\end{array}$ & 1 & $\begin{array}{l}\text { Intense, mixed, } \\
\text { intra- and subepithelial }\end{array}$ & 1 & Missing \\
\hline 2 & $\begin{array}{l}\text { Partially persistent } \\
\text { defect }\end{array}$ & 2 & Mature fibrous tissue & 2 & $\begin{array}{l}\text { Moderate, mixed, } \\
\text { intra- and subepithelial }\end{array}$ & 2 & Present \\
\hline 3 & $\begin{array}{l}\text { Complete } \\
\text { epithelialization }\end{array}$ & & & 3 & Minimal, sparse & & \\
\hline
\end{tabular}

Scheme used for semi-quantitative evaluation of the level of epithelialization, quality of connective tissue, and presence of inflammatory infiltrates and reactive osteoplasia.

overnight and finally stored at $-70{ }^{\circ} \mathrm{C}$ until RNA isolation. Prior to RNA isolation, the tissues were homogenized in TRI Reagent RT (Molecular Research Center, Inc., USA) with zirconia/silica beads using a MagNALyser instrument (Roche, Switzerland). RNA was obtained by phase separation using 4-bromoanisole with 15 min of centrifugation at $4{ }^{\circ} \mathrm{C}$ and was further purified using an RNeasy Kit (Qiagen, Germany) according to the manufacturers' instructions for an animal tissue protocol. A sample of the obtained total RNA in $18 \mu \mathrm{L}$ of Qiagen RNase-free water was spectrophotometrically measured to determine the concentration and purity in a NanoDrop $2000 \mathrm{UV}-\mathrm{V}$ is spectrophotometer (Thermo Fisher Scientific, Waltham, USA). The same amount of RNA from every sample $(2.4 \mu \mathrm{g})$ was directly reverse transcribed using M-MLV reverse transcriptase (200 U, Invitrogen) and an oligo(dT) RT primer at $37^{\circ} \mathrm{C}$ for $1.5 \mathrm{~h}$. Then, the cDNA was stored at $-20{ }^{\circ} \mathrm{C}$ until use. RNA integrity was checked by means of electrophoresis.

\section{qPCR}

For qPCR purposes, the cDNA of each sample was prepared in triplicate reactions in a 384-well plate with the assistance of a Nanodrop II liquid dispenser (Innovadyne Technologies, Rohnert Park, CA). Each reaction mixture with a total volume of $3 \mu \mathrm{L}$ consisted of $0.5 \mu \mathrm{L}$ of 5-fold diluted cDNA, 10 pmol of a gene-specific primer pair (Generi Biotech, Hradec Kralove, Czech Republic, Supplement S1) and 1.5 $\mu \mathrm{L}$ of master mix (QuantiTect SYBR Green PCR Kit, Qiagen, Hilden, Germany) following the manufacturer's recommendations. qPCR was performed in a LightCycler 480 (Roche Applied Science, https://www.roche.com/) under the following conditions: denaturation $\left(95^{\circ} \mathrm{C}\right.$ for $15 \mathrm{~min}$ ) followed by 45 amplification cycles $\left(95^{\circ} \mathrm{C}\right.$ for $15 \mathrm{~s}, 58{ }^{\circ} \mathrm{C}$ for $30 \mathrm{~s}$ and $72{ }^{\circ} \mathrm{C}$ for $30 \mathrm{~s})$, and the quantification cycle $(\mathrm{Cq})$ value was obtained for each gene in each reaction. The gene expression of 9 target genes and 3 housekeeping genes (HPRT-1, GAPDH, HMBS), as potential reference genes for the normalization of data (Supplement S8), was measured. Using the Excel add-in software NormFinder, HPRT-1 was evaluated as the reference gene showing minimal gene expression among all samples tested. HPRT-1 also served as a positive internal control for qPCR. Specific primer pairs were designed using the Primer-BLAST NCBI web page. To prevent the detection of genomic DNA, residual primers were designed to ensure that at least one primer of a given primer pair spanned an mRNA exon junction. Gene expression was calculated using the formula $\left[1 /\left(2^{\text {Cqoftarget gene }}\right)\right] /\left[1 /\left(2^{\text {Cqofreferencegene }}\right)\right]$ ( ref. $\left.^{19}\right)$, including adjustment to reference gene expression. Relative gene expression was characterized as the ratio of the gene expression of the sample under the tested experimental treatment to that of the control sample without experimental treatment. Melting curve analysis of the amplified products was performed to test product specificity using LightCycler 480 Software 1.5.0.39 (Roche Applied Science; https://www.roche.com), and negative controls were included during the entire process to monitor potential nucleic acid contamination.

\section{Statistical analyses}

Because we had no prior information of possible measurement results, we used the resource equation ${ }^{20}$ to estimate the number of animals. The optimal number of investigational units for a simple analysis of variance (ANOVA) design assumes the error degrees of freedom (Error $\mathrm{df}=$ total $\mathrm{df}$ - treatment $\mathrm{df}$ ) to be 10 to 20 . In our experiment, there were three treatments investigated, and the animals were sacrificed at three time points. Each animal underwent two investigational procedures. Thus, a total 24 animals yield the Error df of 14 (24 animals yield 48 investigational units, 16 for each time point resulting in $14 \mathrm{EDF}$ given three treatments).

PPD, CAL and bone loss were evaluated using oneway ANOVA. Differences between the initial value (Day 0) and the second measurement (2 weeks, 6 weeks, or 12 weeks) were tested separately for each defect, and summaries of the results are displayed as error bar plots (Fig. 3 and 4). Histological variables were analysed on a semi-quantitative ordinal scale, and the results are shown in bar plots. Differences between the analysed groups were tested using the chi-square test (Supplements S1S4). Molecular data were logarithmically transformed to achieve an approximately normal distribution. Transformed molecular data were analysed using ANOVA and displayed in error bar plots. Analyses were performed using R 3.4.3 software $^{21}$. The level of significance was considered alpha $=0.05$. 


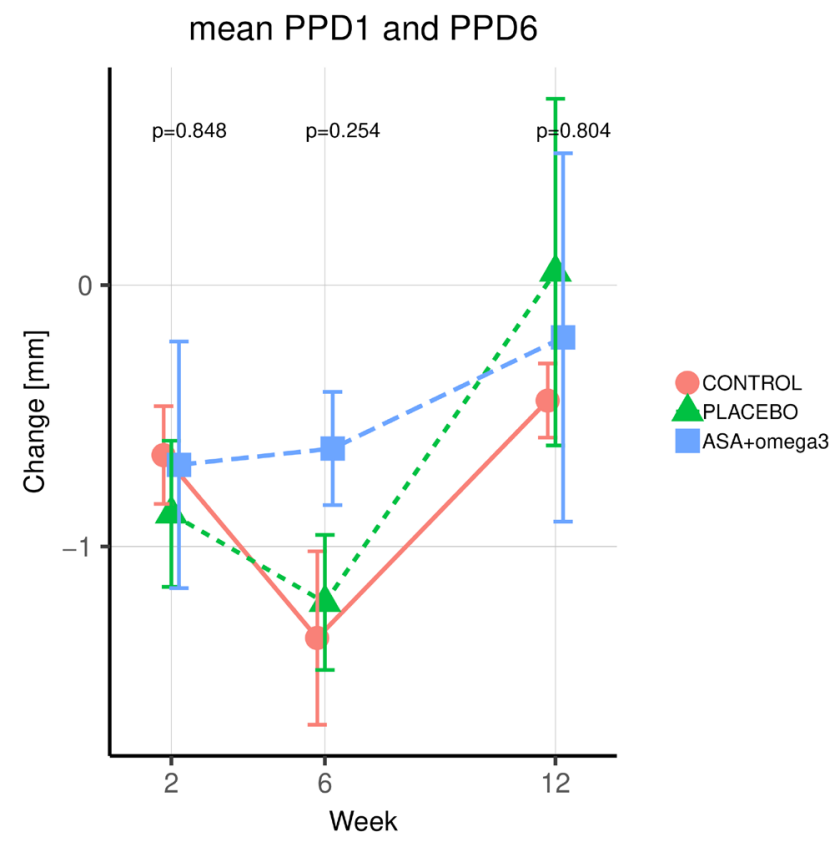

Fig. 3. Probing pocket depth.

Difference between baseline measurements (Day 0) of PPD at locations 1 and 6 and PPD measured at 2 weeks, 6 weeks, or 12 weeks.

\section{RESULTS}

No significant differences were found between groups at the measured time points. The changes in variables are described in detail below.

At two weeks after the surgery, in all groups, PPD and CAL showed insignificant decline compared to the initial value (Day 0) (Fig 3 and 4). A difference in CAL change between the groups can only be seen for locations 2 and $5(P=0.041)$. No other differences were observed between the groups regarding PPD and CAL (Fig 3 and 4). No significant differences were found for the molecular indicators or histopathological criteria (Supplements S1-S5).

At six weeks, according to a still-declining PPD, the defects tended to heal, or post-operative swelling persisted. At the same time point, however, CAL increased in the ASA+omega 3 and PLACEBO groups, in contrast to the decrease in CAL in the CONTROL group. Nevertheless, no significant differences in the changes in PPD and CAL were observed between the groups (Fig 3 and 4). However, there was a significant difference in IL-17 levels $(P=0.05)$ in the examined tissues, with the highest values being observed in the PLACEBO group (Supplement S6i). For the other molecular and histopathological parameters, no differences could be confirmed at this time point.

At twelve weeks, both PPD and CAL returned to levels close to baseline in all study groups. Indeed, PPD and CAL almost reached baseline values (Day 0) at closer measurement locations ( 1 and 6 ), nut there was no significant difference between the groups (Fig 3 and 4). The examined molecular markers did not show any difference between groups at this time point (Supplement S6).

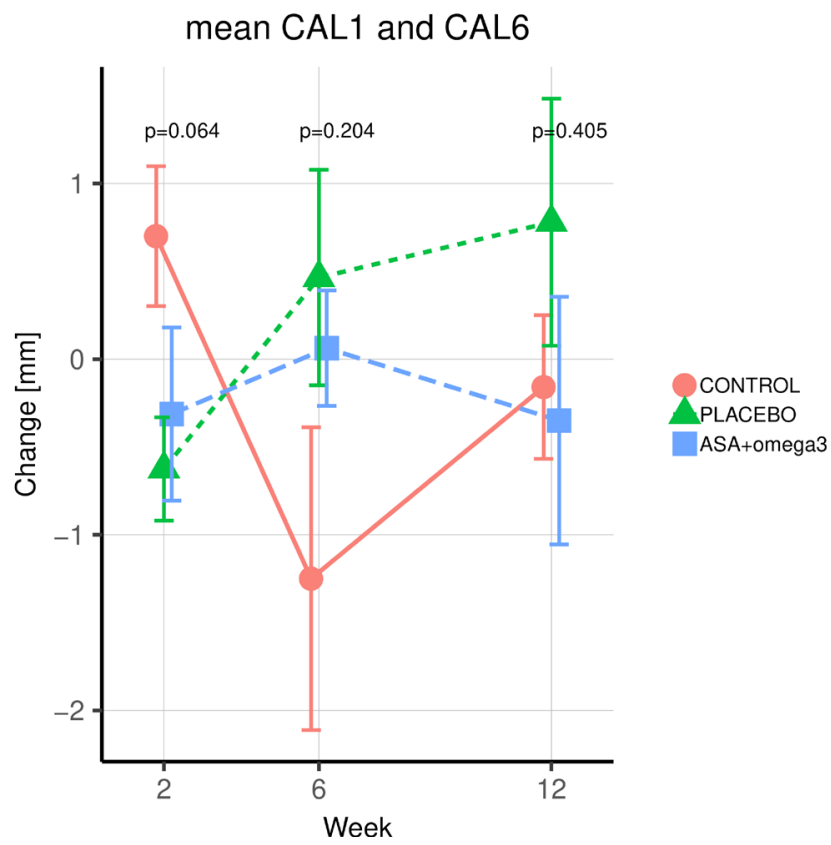

Fig. 4. Clinical attachment level.

Difference between baseline measurements (Day 0) of CAL at locations 1 and 6 and PPD measured at 2 weeks, 6 weeks, or 12 weeks.

However, the difference in histologically evaluated bone loss was found to be nearly significant $(P=0.057)$, and the observed bone loss was greater in the PLACEBO group compared to the other study groups (Supplement S5). In all the groups, bone loss was greater than at baseline.

During the observation period of 12 weeks, tissue levels of FGF-7, IL-1 $\beta$ and TIMP-1 showed a statistically significant decrease over time (Supplement S7). This was not the case for IL-10, IL-17, MMP-9, TGFß-1 and VEGF, whose levels tended to remain constant, or for TNF- $\alpha$, which increased insignificantly.

Of the histopathological parameters, changes could be observed in terms of an increasing grade of epithelialization, maturation of connective tissue, and decline of inflammatory infiltration (Supplements S1-S4). However, the results did not prove to be significant.

The defects at different healing stages with their histological characteristics are shown in Fig 5.

\section{DISCUSSION}

In this study, we analysed the clinical, histopathological and molecular biological parameters of the healing of periodontal defects after treatment using three different approaches. We used the ligature-induced periodontitis model in rabbit.

The primary observed parameters, PPD and CAL, showed rather contradictory results and rarely reached the level of statistical significance. The measured PPD values at 2 weeks showed a decline, meaning that the defect was smaller than at Day 0. This difference might 

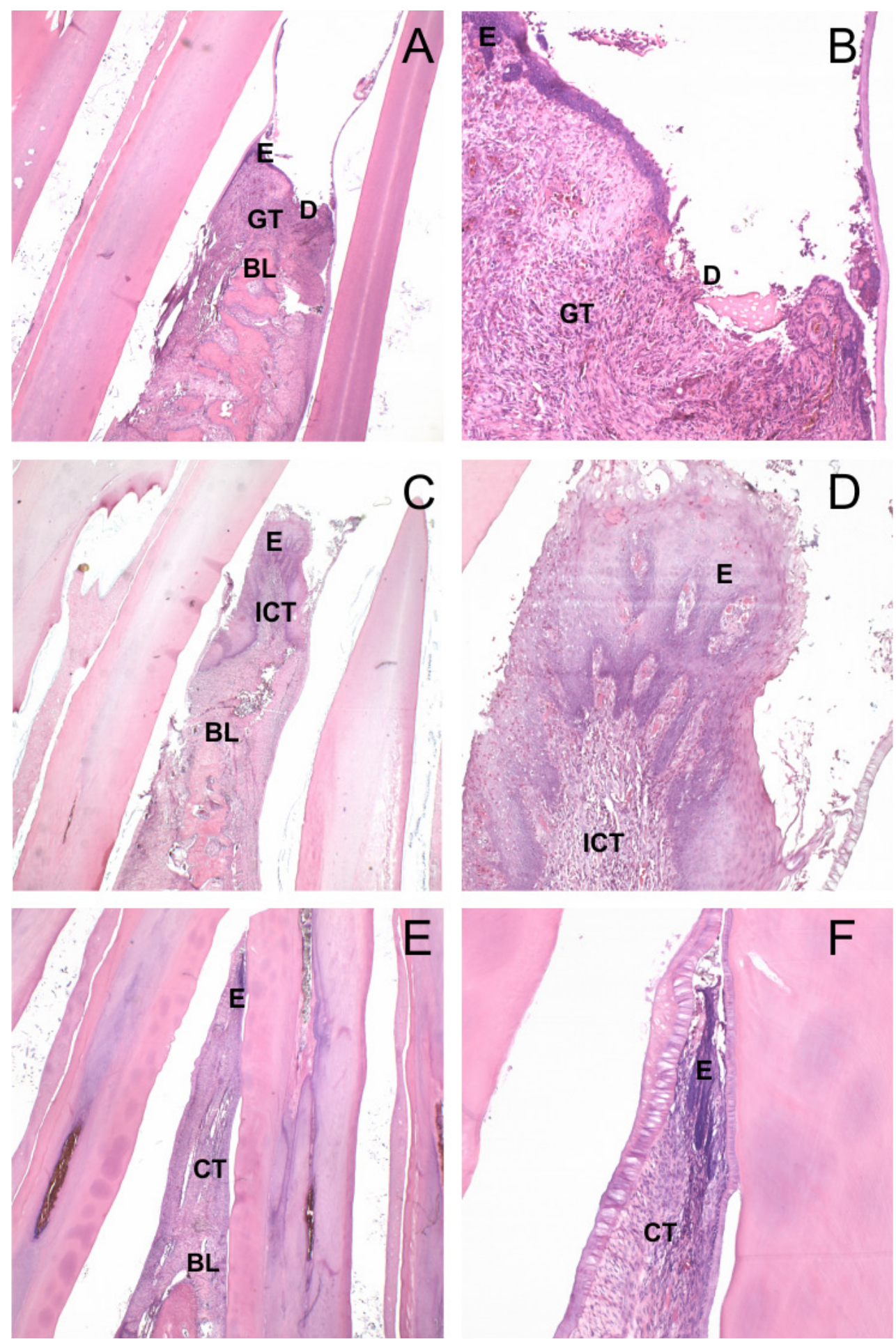

Fig. 5. Defect healing stages and histological characteristics.

At 2 weeks (A,B), 6 weeks (C,D), and 12 weeks (E,F) after surgery (H\&E, original magnification x20 (A,C,E), and x100 (B,D,F). (A,B) Two weeks after surgery, the defect persists, with epithelialization only observed at its periphery, and dense mixed inflammatory infiltrate is present at the base of the wound and within the highly cellular granulation tissue. (C,D) Six weeks after surgery, the defect is epithelialized, with moderate to intense mixed inflammatory infiltrates within the epithelium and the underlying highly cellular immature connective tissue, and proliferation of fibroblasts and capillaries is observed. (E,F) Twelve weeks after surgery, the defect is completely epithelialized, with minimal subepithelial inflammatory infiltrate, and mature fibrous tissue is present in the interdental area. E: epithelium; D: defect; GT: granulation tissue; ICT: immature connective tissue; CT: connective tissue; BL: bone level. 
have been caused by oedema rather than healing of the site. At 6 weeks, a negative difference was even more noticeable, meaning that healed or still swollen tissues impeded the access of the probe to greater depth. For the ASA+omega3 group, this deviation was less obvious, probably due to less post-operative oedema in this group. However, at 12 weeks, PPD increased again as oedema subsided and immature attachment allowed the probe to penetrate deeper. At this point, PPD almost reached the initial values measured at day 0 .

On the other hand, CAL at 2 weeks corresponded to the initial values in the ASA+omega 3 and PLACEBO groups, but not in the CONTROL group, where CAL increased. At 6 weeks, the opposite situation was observed, when the values increased above the initial levels in the ASA+omega 3 and PLACEBO groups, but a decline in CAL was observed in the CONTROL group. We can attribute this finding to the post-operative oedema, which was similarly noticeable in the PPD values. Finally, at 12 weeks, the CAL values for all the groups returned to near-baseline levels. Thus, the levels of CAL at 12 weeks did not differ from the initial measurements, showing no significant change in attachment position after the observation period.

It is important to note when considering our results that the artificial defects were created with an average PPD of $2.6 \mathrm{~mm}$ and CAL of $5.6 \mathrm{~mm}$ (locations 1 and 6 ), and histological bone loss could not be examined on day 0 . The PPD and CAL varied by approximately $1 \mathrm{~mm}$. The standard deviations of the PPD and CAL changes were $1.1 \mathrm{~mm}$ and $1.5 \mathrm{~mm}$, respectively. Using a periodontal probe, the discriminating power is $1 \mathrm{~mm}$, so an experienced observer can measure differences with a $0.5 \mathrm{~mm}$ accuracy at best. All the above-mentioned facts imply that we were dealing with relatively small changes in the evaluated data compared to large data variability and measurement uncertainty, which could be responsible for the non-significant results. Other similar studies performed in a ligature-induced periodontitis model have never used PPD and CAL as primary outcomes. Instead, bone $\operatorname{loss}^{17,22-25}$ or root exposure ${ }^{24}$ have predominantly been measured clinically with a $0.5 \mathrm{~mm}$ scaled probe after defleshing of necropsies or have been histologically measured in stained samples. These studies use rats or rabbits for their experiments. Or, if PPD was selected as a primary criterion, a larger animal such as monkey was employed ${ }^{26,27}$. However, for our experiment, clinical parameters PPD and CAL were of major interest.

Gradual decrease in the tissue levels of FGF-7 (also known as keratinocyte growth factor), IL-1 $\beta$ (pro-inflammatory mediator) and TIMP-1 (metalloproteinase inhibitor) corresponded to the healing of defects. It may be of interest that MMP9 was maintained at a significant level even 12 weeks after ligature removal, when tissue remodelling is assumed to be complete.

Our approach includes the induction of pro-resolution pathways by aspirin and omega-3 PUFAs. Such interventions in GT are assumed to oppose the pro-inflammatory phenotype of GT and result in better and faster healing outcomes. Therefore, there might be important benefits for healing in preserving and experimentally manipulating GT in periodontal surgery. In the present study, we were not able to prove such effect. Nevertheless, the enhanced granulation tissue did not impair healing outcomes.

\section{CONCLUSION}

In this experimental rabbit model of ligature-induced periodontitis, PPD and CAL cannot be used as primary observed criteria. A trend to earlier healing was observed when a periodontal defect was treated with GT soaked with aspirin and omega-3 PUFAs. However, 12 weeks after the intervention, the three groups did not exhibit any significant differences. We can conclude that the enriched GT may be returned into a periodontal defect with similar treatment outcomes observed in the standard removal of GT with periodontal surgery techniques. Our results should be confirmed and further examined in a subsequent clinical trial.

Acknowledgement: The study was supported by the Ministry of Health of the Czech Republic (Project No. NV16-28462A).

Author contributions: JV, LIH: conceptualization; FH, MK: data curation; FH, MF, MV, MH: formal analysis; JV, LIH: funding acquisition, project administration; $\mathrm{FH}$, JV, EG, MH: investigation; JV, FH: methodology; LIH: resources, supervision; MK: software; FH, LIH: validation; FH: visualization; FH, MV, MH, MK: manuscript writing; LIH, EG, MF: writing - review and editing.

Conflict of interest statement: None declared.

\section{REFERENCES}

1. Lindhe J, Lang NP, Karring T. Clinical periodontology and implant dentistry. 5th ed. Blackwell Munksgaard; 2008.

2. Lang NP, Lindhe J, Berglundh T, Giannobile WV, Sanz M. Clinical periodontology and implant dentistry. 6th ed. Wiley-Blackwell; 2015.

3. Serhan CN, Brain SD, Buckley CD, Gilroy DW, Haslett C, O'Neill LA, Perretti M, Rossi AG, Wallace JL. Resolution of inflammation: state of the art, definitions and terms. FASEB J 2007;21(2):325-32.

4. Van Dyke TE. Control of inflammation and periodontitis. Periodontol 2000 2007;45(1):158-66.

5. Kurgan Ş, Kantarci A. Molecular basis for immunohistochemical and inflammatory changes during progression of gingivitis to periodontitis. Periodontol 2000 2018;76(1):51-67

6. Freire MO, Van Dyke TE. Natural resolution of inflammation. Periodontol 2000 2013;63(1):49-164.

7. Weylandt KH, Chiu C-Y, Gomolka B, Waechter SF, Wiedenmann B. Omega-3 fatty acids and their lipid mediators: Towards an understanding of resolvin and protectin formation. Prostaglandins Other Lipid Mediat 2012;97(3/4):73-82.

8. Serhan CN. A search for endogenous mechanisms of anti-inflammation uncovers novel chemical mediators: missing links to resolution. Histochem Cell Biol 2004;122(4):305-21.

9. Chiang N, Arita M, Serhan CN. Anti-inflammatory circuitry: lipoxin, aspirin-triggered lipoxins and their receptor ALX. Prostaglandins Leukot Essent Fatty Acids 2005;73(3-4):163-77.

10. Serhan CN, Clish CB, Brannon J, Colgan SP, Chiang N, Gronert K. Novel functional sets of lipid-derived mediators with antiinflammatory actions generated from omega- 3 fatty acids via cyclooxygenase 2-nonsteroidal antiinflammatory drugs and transcellular processing. J Exp Med 2000;192(8):1197-204.

11. Serhan CN1, Hong S, Gronert K, Colgan SP, Devchand PR, Mirick 
G, Moussignac RL. Resolvins: a family of bioactive products of omega-3 fatty acid transformation circuits initiated by aspirin treatment that counter proinflammation signals. J Exp Med 2002;196(8):1025 37

12. Hasturk $H$, Kantarci $A$. Activation and resolution of periodontal inflammation and its systemic impact. Periodontol 2000 2015;69(1):255-73.

13. Ge S, Mrozik KM, Menicanin D, Gronthos S, Bartold PM. Isolation and characterization of mesenchymal stem cell-like cells from healthy and inflamed gingival tissue: potential use for clinical therapy. Regen Med 2012;7(6):819-32.

14. Apatzidou DA, Nile C, Bakopoulou A, Konstantinidis A, Lappin DF. Stem cell-like populations and immunoregulatory molecules in periodontal granulation tissue. J Periodontal Res 2018;53(4):610-21.

15. Larjava $\mathrm{H}$ (ed.). Oral Wound Healing. Wiley-Blackwell; 2012.

16. Kilkenny C, Browne W, Cuthill IC, Emerson M, Altman DG. Anima research: reporting in vivo experiments: the ARRIVE guidelines. $\mathrm{Br} J$ Pharmacol 2010;160(7):1577-9.

17. Yang H, Aprecio RM, Zhou X, Wang Q, Zhang W, Ding Y, Li Y. Therapeutic effect of TSG- 6 engineered iPSC-derived MSCs on experimental periodontitis in rats: a pilot study. PLoS One 2014;9(6):e100285-e100285.

18. Van Dyke TE. Pro-resolving mediators in the regulation of periodontal disease. Mol Aspects Med 2017;58:21-36.

19. Zelnickova P, Matiasovic J, Pavlova B, Kudlackova H, Kovaru F, Faldyna M. Quantitative nitric oxide production by rat, bovine and porcine macrophages. Nitric Oxide 2008;19(1):36-41.

20. Mead R. The design of experiments: statistical principles for practical applications. 1st ed.; Cambridge University Press; 1990
21. R Core Team. R: A Language and Environment for Statistical Computing. R Foundation for Statistical Computing; Vienna, 2013.

22. Botelho MA, Martins JG, Ruela RS, I R, Santos JA, Soares JB, França MC, Montenegro D, Ruela WS, Barros LP, Queiroz DB, Araujo RS, Sampio FC. Protective effect of locally applied carvacrol gel on ligature-induced periodontitis in rats: a tapping mode AFM study. Phytother Res 2009;23(10):1439-48

23. Botelho MA, Martins JG, Ruela RS, Queiroz DB, Ruela WS. Nanotechnology in ligature-induced periodontitis: protective effect of a doxycycline gel with nanoparticules. J Appl Oral Sci 2010;18(4):335-42.

24. Lee CT, Teles R, Kantarci A, Chen T, McCafferty J, Starr JR, Brito LC, Paster BJ, Van Dyke TE. Resolvin E1 Reverses Experimental Periodontitis and Dysbiosis. J Immunol 2016;197(7):2796-806.

25. Serhan CN, Jain A, Marleau S, Clish C, Kantarci A, Behbehani B, Colgan SP, Stahl GL, Merched A, Petasis NA, Chan L, Van Dyke TE. Reduced inflammation and tissue damage in transgenic rabbits overexpressing 15-lipoxygenase and endogenous anti-inflammatory lipid mediators. J Immunol 2003;171(12):6856-65.

26. Hussain MZ, Ghani QP, Zhang JC, Enriquez B, Hayashi C, Wirthlin MR. Alterations of fibroblast metabolism in early ligature-induced periodontitis in the cynomolgus monkey. J Periodontol 1994;65(8):771-

27. Wirthlin MR, Hussain MZ. Clinical and light microscopic observations of gingivitis and early ligature-induced periodontitis in the cynomolgus monkey. J Periodontol 1992;63(6):533-9.

Supplemental Material: The online version of this article (doi: $10.5507 /$ bp.2020.003) offers supplemental material. 